\title{
Using Cuba's example to improve primary healthcare in Canada
}

\author{
Jae-Yung Kwon and Wendy Masinde
}

\section{University of British Columbia}

The Alma-Ata Declaration in 1978 encouraged many countries to incorporate primary healthcare (PHC) principles. It was the first time that all World Health Organization (WHO) members recognized that access to basic health services was a human right, in what was known as "Health for All by the Year 2000." ${ }^{1}$ PHC refers to services that prevent illness and promote health by addressing housing, education, income, and the environment. ${ }^{2}$ In Canada, the principles of PHC include 1) making health services universally available, 2 ) encouraging people to make decisions regarding their own health, 3) promoting health through education, sanitation, maternale and childcare, 4) having appropriate technology that is adaptable to local needs, and 5) maintaining inter-sectoral cooperation through involvement with other sectors such as agriculture, education, and housing. ${ }^{2}$ Although PHC played a key role in reforming health services and changing attitudes from treatment to prevention of diseases, some have questioned whether PHC was even successful because the general health status of the population was still poor in many developing countries. ${ }^{1}$ Unfortunately, the lack of understanding of the importance of $\mathrm{PHC}$ principles on health undermined the translation of $\mathrm{PHC}$ principles into tangible reforms, ${ }^{3}$ with many countries diverting its focus to acute hospital care. ${ }^{1}$ These trends have not only led to decreased access to primary healthcare services; they have also made it difficult to meet the changing social patterns related to health including poverty, hunger and chronic illnesses. ${ }^{3}$

Although the Canadian healthcare system is based on PHC principles, Canada is not immune to its focus on acute hospital care. According to the National Health Expenditure Trends, Canada's health spending in 2013 reached \$211 billion with $30 \%$ of total spending on acute hospitals, $16.3 \%$ in drugs, $15 \%$ in physician compensations, and only $5 \%$ for public health which includes food and water safety, health education and immunization programs. ${ }^{5}$ Unfortunately, the priorities shifted towards acute hospital care as the technological capacity of clinical medicine grew. ${ }^{10}$ In addition, there have been inconsistent health care goals among Canadians, with some seeing it as increased access to acute care hospitals, the reduction of waiting times, or how many people are serviced. ${ }^{6}$ As a result, having a common public health priority is important to prevent people from being ill, reduce the cost on acute hospitals and improve the quality of life for Canadians. ${ }^{10}$

Canada could look towards Cuba for help. For example, Cuba demonstrates strong, consistent values toward primary healthcare, and has transformed these values into tangible reforms. Despite decades of severe economic embargo and difficulty, Cuba was not only able to maintain a strong PHC system, but also achieve impressive health indices comparable to those in more developed countries. ${ }^{7}$ This was evidenced by infant mortality rates of 4.76 deaths $/ 1000$ live births (important indicator for the level of health in a country) comparable to those in developed countries including the US [5.9] and Canada [4.78]. In contrast, countries with levels of economic performance similar to Cuba had much higher rates, such as Bolivia [39.76], Honduras [19.28] and Ecuador [18.48]. ${ }^{8}$ Other health indicators such as life expectancy shared a similar trend that was favourable to Cuba. ${ }^{7}$ However, one of the most important factors cited for Cuba's health achievements for such a poor country was their strong emphasis from treatment services for the few to disease prevention and health promotion for the entire population. ${ }^{8}$ The most revolutionary idea of the Cuban system is the development of community-based clinics in every neighbourhood, where physicians and nurses live in the community that they serve. ${ }^{9}$ These clinics not only provide a holistic, family and community-centered care but also health promotion activities which include teaching about nutrition, hygiene, and environmental issues. They also help to organize grandparents' circles for elders, 
and related activities for teens. ${ }^{7}$ Rather than focusing on diagnosis and treatment, the physicians and nurses help to address non-medical determinants of health and create a health culture within their communities. ${ }^{7}$ As a result, Cuba's priority towards community-based clinics was one of the key factors in producing impressive health results.

Although the aim of the PHC principles was to address a wide range of factors that prevent disease and promote health, the ways in which primary healthcare services are organized, delivered, and funded in Canada have focused on a narrow scope of acute hospital-based care. To increase access to primary healthcare services and meet the changing social patterns related to health, we must revisit how our funding structure and health goals address the PHC principles. Once we realize that health is not just about treating disease, we can begin to learn from Cuba's example of community-based clinics and truly improve primary healthcare in Canada.

\section{References}

1. Moghadam MN, Sadeghi V, Parva S. Weaknesses and challenges of primary healthcare system in Iran: a review. Int J Health Plann Manage. 2012; 27(2):e121-e131.

2. Health Canada. About primary health care [internet]. Ottawa, Health Canada. [last update 2012 Aug 23; cited 2014 Apr 23]. Available from: http://www.hc-sc.gc.ca/hcs-sss/prim/about-apropos-eng.php.

3. World Health Organization. Introduction and Overview [internet]. Geneva, World Health Organization. [last update 2008 Jan 1; cited 2014 Apr 23]. Available from: http://www.who.int/whr/2008/overview/en/index.html.

4. Duncan S, Reutter L. A critical policy analysis of an emerging agenda for home care in one Canadian province. Health Soc Care Community. 2006; 14(3):242-53.

5. Canadian Institute for Health Information (ClHI). National health expenditure trends, 1975 to 2013 [internet]. Ottawa, CIHI. [last update 2013 Oct 29; cited 2014 Apr 23]. Available from: https://secure.cihi.ca/ estore/productFamily.htm?locale=en\&pf=PFC2400

6. Muzyka D, Hodgson G, Prada G. The inconvenient truths about Canadian health care [internet]. Ottawa, The Conference Board of Canada. [last update 2012 April 29; cited 2014 Apr 23]. Available from: http://www. conferenceboard.ca/cashc/research/2012/inconvenient_truths.aspx

7. Spiegel JM, Yassi A. Lessons from the margins of globalization: appreciating the Cuban health paradox. J Public Health Policy. 2004; 25(1):85-110.

8. Central Intelligence Agency. The world factbook: infant mortality rate [internet]. Langley, Central Intelligence Agency. 2013 [last update 2013 Apri 24; cited 2014 Apr 23]. Available from: https://www.cia.gov/library/ publications/the-world-factbook/fields/2091.html.

9. Fitz D. Why is Cuba's health care system the best model for poor countries? [internet]. Place of publication unknown [last update 2012 July 12; cited 2014 Apr 23]. Available from: http://mrzine.monthlyreview.org/2012/ fitz071212.html.

10. Public Health Agency of Canada. The role and organization of public health [internet]. Ottawa, Public Health Agency of Canada. [last update 2011 Nov 08; cited 2014 Apr 23]. Available from: http://www.phac-aspc.gc.ca/ publicat/sars-sras/naylor/3-eng.php.
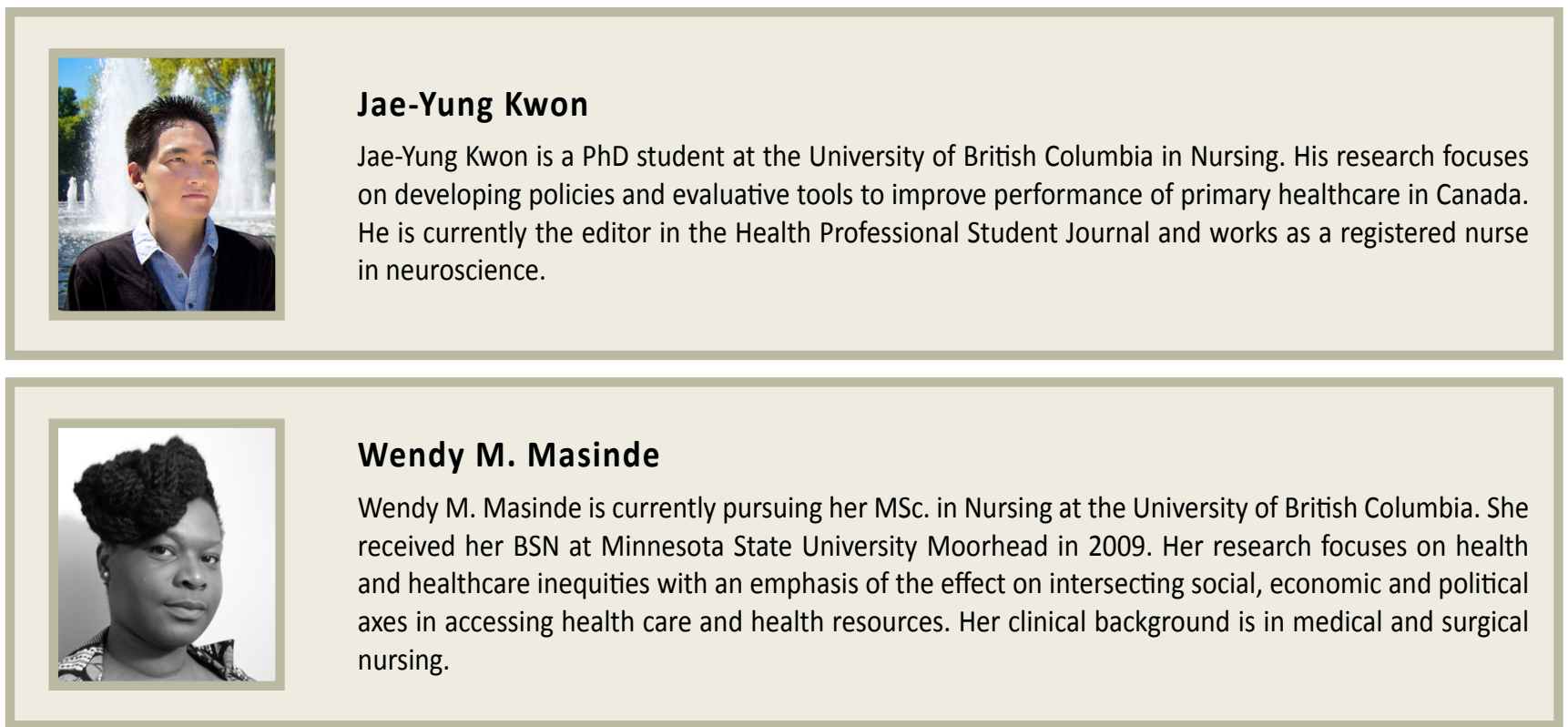